\title{
Loss characterization in micro-cavities using the thermal bistability effect
}

\author{
H. Rokhsari, S. M. Spillane, K. J. Vahala \\ Department of Applied Physics, California Institute of Technology, Pasadena, California 91125 \\ rokhsari@caltech.edu
}

\begin{abstract}
We investigate the role of absorption and scattering losses in limiting the quality factor of toroidal microcavities by monitoring the threshold power for thermal bistability in these structures. (C)2004 Optical Society of America

OCIS codes: 230.4320 Nonlinear optical devices; 140.4780 Optical resonators; 190.1450 Bistability; 120.6810 Thermal effects
\end{abstract}

At a sufficiently high $\mathrm{Q}$, whispering gallery mode micro-resonators can enter a regime where minute injected optical powers can result in large thermal nonlinearities [1,2]. The circulating intensity in these cavities, greatly enhanced due to their high quality factors and small mode volumes, can generate substantial heat, and the resulting temperature change can produce thermal bistability [3]. In this work we exploit this phenomenon as a new tool for characterizing distinct optical loss mechanisms responsible for limiting the quality factor of high- $Q$ toroidal micro-cavities [4]. The results provide insight into the relative importance of surface scattering and absorption centers in these structures.

Thermal broadening/compression of the resonance line shape is frequently encountered in Ultra-HighQuality (UHQ) micro-cavities $\left(\mathrm{Q}>10^{8}\right)$ [5]. As the laser frequency is swept across the cavity resonance, optical power coupled into the resonator is partially absorbed and converted to heat, hence altering the optical properties of the bulk medium and shifting the resonant frequency either along or opposite to the direction of laser scanning. The dominant effect is due to the temperature dependent refractive index of the cavity material which in the case of silica results in a negative frequency shift of the resonance with increased temperature. This frequency shift is proportional to the injected power to the optical mode:

$$
\left(v-v_{0}\right) / \Delta v=-P_{i n} / P_{t h}
$$

where threshold power $\left(P_{t h}\right)$ here is defined as the power required to shift a resonance by its width. Threshold power is inversely proportional to the quality factor and to the absorption fraction of lost power (i.e., power lost to absorption relative to total power lost through all mechanisms contributing to intrinsic Q). In cases of exceptionallysmooth whispering-gallery surfaces (i.e., low scattering loss) and larger diameter resonators (not whispering gallery loss limited) the absorption fraction can approach unity and a $Q^{-1}$ behavior of threshold power is expected. On the other hand, if non-thermal losses (scattering or WGM losses) are the dominant loss mechanism, the absorption fraction is proportional to $\mathrm{Q}$ and a $\mathrm{Q}^{-2}$ dependence in threshold power should be observable in modes belonging to the same resonator, but having different quality factors.

Figure 1 shows the measured thermal shift of the resonant frequency vs. input power for a high $Q$ $\left(Q=0.9 \times 10^{8}\right)$ whispering gallery mode in a toroid micro-resonator at two different wavelengths. Although $Q$ 's at these two frequencies are about the same, the threshold power at $970 \mathrm{~nm}$ is a factor of 20 higher than that at 1545 $\mathrm{nm}$. The above difference suggests a higher absorptive loss at $1545 \mathrm{~nm}$ compared to $970 \mathrm{~nm}$ which can not be explained in terms of silica absorption as fused silica is about 4 times more absorptive at 970 nm. However assuming that there is a monolayer of water molecules on the resonator surface [6,7] and that an efficient heat transfer mechanism from surface water to the bulk glass exists, a difference in power threshold of about 25 can be predicted. This is based on our absorption loss calculations for fundamental modes of a sphere with a similar diameter and is in good agreement with the empirical value of 20 from figure 1 . The substantial difference in absorption losses at these frequencies and yet the nearly equal quality factors suggest that surface scattering is the dominant loss mechanism in this micro-toroid.

Fig. 2 shows the measured threshold powers for different modes of the same micro-toroid (resonator 1). Close to quadratic dependence of threshold power on $Q$ confirms our prior conclusion that almost all the losses in 
this sample are scattering losses. The same experiment on a different sample (resonator 2) however, reveals an almost linear behavior that attests the quality factor in this case is absorption limited (better surface quality).

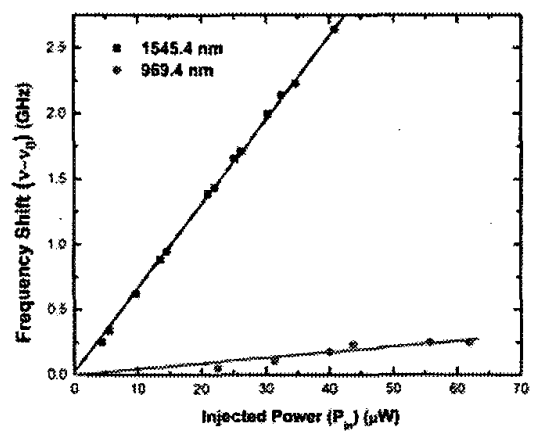

Fig. 1. Thermal shift of the resonant frequency of a whispering gallery mode in a toroid micro-cavity as a function of coupled power to the resonator. The squares (blue) are the data at wavelength $1545.4 \mathrm{~nm}$ and the circles (red) are for the same fundamental mode at $969.4 \mathrm{~nm}$. The bistability threshold power is higher by a factor of 20 at $969.4 \mathrm{~nm}$ and is believed to be due to lower absorption of water and glass at this wavelength.

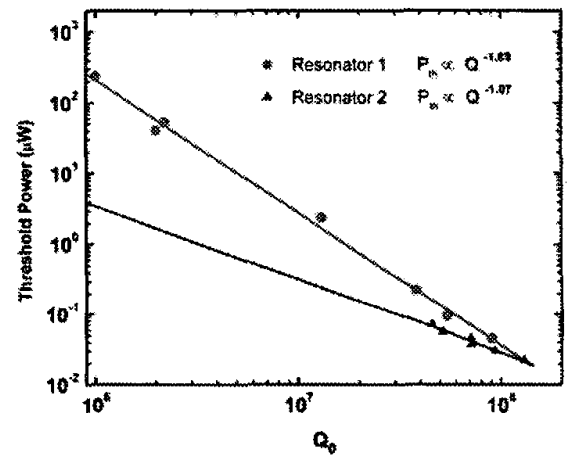

Fig. 2. Thermal bistabilty threshold power plotted versus quality factor for different optical modes of two toroidal microresonators. Circles (red) show a quadratic dependence of the threshold power on the quality factor of the optical mode in the first resonator, while for the second resonator shown in triangles (blue) the dependence is linear.

This demonstrates that studying nonlinear thermal effects is a good method for characterizing different loss mechanisms in micro-resonators and provides valuable information about the surface contaminants. As a result, real time monitoring of these properties can potentially be used to study the dynamics of interaction between the resonator surface and its environment.

\section{References}

[1] F. Treussart et al., "Evidence for intrinsic Kerr bistability of high-Q microsphere resonators in superfluid helium" The European Physical Journal D 1, 235-238 (1998).

[2] V.B Braginsky, M. L. Gorodetsky and V.S. Ilchenko, "Quality-factor and nonlinear properties of optical whispering gallery modes" Physics Letters A 137, 393-397 (1989).

[3] V. S. Ilchenko and M. L. Gorodetsky, "Thermal Nonlinear Effects in Optical Whispering Gallery Microresonators" Laser Physics 2, 10041009 (1992).

[4] D. K. Armani, T. J. Kippenberg, S. M. Spillane, and K. J. Vahala, "Ultra-high-Q toroid microcavity on a chip," Nature 421, 925-

$928(2003)$.

[5] L. Collot, V. Lefevre-Seguin, M. Brune, J. M. Raimond and S. Harche, "Very High-Q Whispering-Gallery Mode Resonances Observed on Fused Silica Microspheres" Europhysics Letters 23, 327-334 (1993).

[6] M. L. Gorodetsky, A. A. Savchenkov and V.S. Ilchenko, "Ultimate $Q$ of optical microsphere resonators" Optics Letters 21, 453-455 (1996).

[7] D. W. Vernooy, V. S. Iichenko, H. Mabuchi, E. W. Streed, and H. J. Kimble, "High-Q measurements of fused-silica microspheres in the near infrared" Optics Letters 23, 247-249 (1998). 